\title{
Optical solitons in fibre Bragg gratings with third- and fourth- order dispersive reflectivities
}

\author{
${ }^{1}$ Yakup Yildırım, ${ }^{2,3,4,5}$ Anjan Biswas, ${ }^{5}$ Padmaja Guggilla, ${ }^{5}$ Salam Khan, \\ ${ }^{3}$ Hashim M. Alshehri and ${ }^{6}$ Milivoj R. Belic \\ ${ }^{1}$ Department of Mathematics, Faculty of Arts and Sciences, Near East University, \\ 99138 Nicosia, Cyprus \\ ${ }^{2}$ Department of Applied Mathematics, National Research Nuclear University, 31 \\ Kashirskoe Hwy, Moscow-115409, Russian Federation \\ ${ }^{3}$ Mathematical Modeling and Applied Computation (MMAC) Research Group, \\ Department of Mathematics, King Abdulaziz University, Jeddah-21589, Saudi Arabia \\ ${ }^{4}$ Department of Mathematics and Applied Mathematics, Sefako Makgatho Health \\ Sciences University, Medunsa-0204, Pretoria, South Africa \\ ${ }^{5}$ Department of Physics, Chemistry and Mathematics, Alabama A\&M University, \\ Normal, AL 35762-4900, USA \\ ${ }^{6}$ Institute of Physics Belgrade, Pregrevica 118, 11080 Zemun, Serbia
}

Received: 22.10 .2021

\begin{abstract}
For the first time in the field of nonlinear optics, we address cubic-quartic solitons appearing in the fibre Bragg gratings with dispersive reflectivity for four different cases of nonlinear refractive-index structures. A complete spectrum of single solitons, together with some straddled solitons, emerges from the integration scheme adopted by us, which is the approach of sine-Gordon equation.
\end{abstract}

Keywords: solitons, Bragg gratings, sine-Gordon equation method

UDC: 532.32

\section{Introduction}

The theory of dynamics of optical solitons has left a lasting impact in telecommunications industry. There are various aspects of soliton science elaborated by the researchers. Soliton switching, intra-channel soliton collisions, quasi-monochromatic soliton dynamics, optical soliton cooling and some other topics are among the main points under interest. Another problem which is not very commonly touched upon is Bragg gratings. This is a very clever remedy to soliton transmission across intercontinental distances when a chromatic dispersion (CD) runs low. In such a crisis situation it is gratings that are introduced in an optical fibre that produces dispersive reflectivity. This ensures a stable propagation of solitons when a necessary delicate balance between dispersion and nonlinearity is maintained.

Bragg gratings have been addressed by many authors [1-26]. The existence and stability of quiescent Bragg-grating solitons have been systematically investigated in a dual-core fibre [1]. The interactions between stable quiescent Bragg-grating solitons in a dual-core system have also been reported in Ref. [2]. The interaction of quiescent gap solitons in coupled fibre Bragg gratings (FBGs) with dispersive reflectivity and cubic-quintic nonlinearity in the both cores has been addressed in Ref. [3]. The authors [4] have proposed and demonstrated experimentally a novel optimally designed FBG-filter scheme, which is based upon single fibres and designed for the conversion from RZ-OOK/DPSK/DQPSK to NRZ-OOK/DPSK/DQPSK format. A novel notchfiltering scheme, which is based on an optimally designed two-degree-of-freedom FBG, has been 
suggested for a transparent all-optical bit-rate NRZ-to-PRZ format conversion [5]. The existence, stability and collision dynamics of moving Bragg-grating solitons in a semi-linear dual-core system have been analyzed in Ref. [6]. The existence and stability of quiescent gap solitons in a system of two linearly coupled Bragg gratings with a cubic-quintic nonlinearity have also been studied [7]. A hybrid-core circularly cladded photonic crystal fibre has been designed and analyzed for applications in the terahertz frequency range [8]. The collisions of moving gap solitons in a system of two identical linearly coupled Bragg gratings with a cubic-quintic nonlinearity have been a subject of the study [9].

Optical solitons in the FBGs with dispersive reflectivity have been retrieved in the earlier studies $[10,11]$. Bright and singular optical soliton solutions for the FBGs with dispersive reflectivity for the parabolic nonlinearity have been recovered by an extended trial-function method [12]. Bright, dark and singular solitons in these FBGs have also been revealed using a method of undetermined coefficients [13]. Optical solitons in the FBGs with dispersive reflectivity have also been obtained by the extended trial-function method for the case of quadratic-cubic nonlinearity [14]. Optical solitons in the mentioned FBGs emerge in the case of cubic-quinticseptic nonlinearity [15]. Using the extended trial function, bright and singular optical solitons have been retrieved in the same FBGs for a parabolic-nonlocal combo nonlinearity [16]. Finally, dark and singular optical solitons in the FBGs with five different forms of nonlinear refractive index have been found using a modified simple equation [17].

A system of partial differential equations for the moving optical solitons in FBGs has been studied in Ref. [18]. As follows from the sine-Gordon equation technique, a complete spectrum of single and straddled solitons emerges in the FBGs with dispersive reflectivity for five different forms of nonlinear refractive index [19]. Chirped and chirp-free solitons in the FBGs having dispersive reflectivity with a parabolic form of nonlinearity can be recovered, using a new Jacobi ellipticfunction expansion [20]. Moreover, the same optical solitons have been revealed in the FBGs with dispersive reflectivity and a quadratic-cubic nonlinearity, basing on a new sub-ODE method [21]. The same sub-ODE approach has yielded in both the chirped and chirp-free optical solitons in the FBGs having dispersive reflectivity and a polynomial form of nonlinearity [22]. Furthermore, optical solitons in the same FBGs revealing the mentioned parabolic-nonlocal combo nonlinearity have been obtained via three prolific integration architectures [23]. Optical solitons in the FBGs with generalized anti-cubic nonlinearity have been retrieved from an extended auxiliary equation [24]. The authors [25] have studied the optical solitons that arise in the FBGs revealing a Kerr law for the refractive index. This is due to an extended Kudryashov's method and a new extended auxiliaryequation approach. Finally, both dark and singular optical solitons appearing in the FBGs have been addressed within the Kudryashov's model in the presence of dispersive reflectivity [26].

At present, another situation comes on board when the CD carries a low count and, hence, a balance between the $\mathrm{CD}$ and the nonlinearity becomes precariously low, being followed by a possible pulse collapse. In such a situation, the CD gets replaced by a combination of third-order and fourth-order dispersions. Thus, with Bragg gratings, the dual dispersion terms introduce socalled third- and fourth-order dispersive reflectivities. In the present study, we introduce the models for four different structural forms of the nonlinear refractive index, where the CD is replaced by the third-order and fourth-order dispersions together with the dispersive reflectivity. The approach of sine-Gordon equation reveals soliton solutions to these four models. A complete spectrum of solitons emerges within this integration scheme, which is discussed in this work. The details will be sketched after a brief introduction into our model. 


\section{Optical solitons}

In this section we address a coupled nonlinear Schrodinger equation for the cases of four different forms for the nonlinear refractive index in FBGs.

\subsection{Kerr law}

The structure of the governing model with the Kerr nonlinear refractive index is given by

$$
\begin{aligned}
& i q_{t}+i a_{1} r_{x x x}+b_{1} r_{x x x x}+\left(c_{1}|q|^{2}+d_{1}|r|^{2}\right) q+i \alpha_{1} q_{x}+\beta_{1} r=0, \\
& i r_{t}+i a_{2} q_{x x x}+b_{2} q_{x x x x}+\left(c_{2}|r|^{2}+d_{2}|q|^{2}\right) r+i \alpha_{2} r_{x}+\beta_{2} q=0,
\end{aligned}
$$

where the complex-valued functions $q(x, t)$ and $r(x, t)$ signify forward- and backward-propagating wave profiles, while $x$ and $t$ are the non-dimensional distance and time in dimensionless form respectively. The first terms reflect a linear temporal evolution, $i=\sqrt{-1}, a_{l}$ and $b_{l}(l=1,2)$ imply the coefficients of respectively the third- and fourth-order dispersions, and $c_{l}$ and $d_{l}$ the coefficients of respectively self-phase modulation (SPM) and cross-phase modulation (XPM). Note also that $a_{l}$ and $\beta_{l}$ imply the coefficients referred respectively to inter-modal dispersion and detuning parameters.

To obtain optical solitons for the FBGs described by Eqs. (1) and (2), we assume the following travelling-wave transformations:

$$
\begin{array}{ll}
q(x, t)=U_{1}(\xi) e^{i \varphi(x, t)}, & r(x, t)=U_{2}(\xi) e^{i \varphi(x, t)}, \\
\xi=x-v t, & \varphi(x, t)=-\kappa x+\omega t+\theta_{0},
\end{array}
$$

where the real-valued functions $\varphi(x, t)$ and $U_{l}(\xi)$ stem respectively from the phase and amplitude components of the soliton, while the real constants $\theta_{0}, \kappa, \omega$ and $v$ denote the phase constant, the wave number, the frequency and the velocity, respectively.

Substituting Eq. (3) into Eqs. (1) and (2), one obtains the real part which is a fourth-order ordinary differential equation (ODE),

$$
U_{\tilde{l}}^{(i v)}+\left(3 \kappa a_{l}-6 \kappa^{2} b_{l}\right) U_{\tilde{l}}^{\prime \prime}+\left(\beta_{l}-\kappa^{3} a_{l}+\kappa^{4} b_{l}\right) U_{\tilde{l}}+c_{l} U_{l}^{3}+d_{l} U_{l} U_{\tilde{l}}^{2}=0,
$$

and the imaginary part which is a third-order ODE,

$$
\left(a_{l}-4 \kappa b_{l}\right) U_{\tilde{l}}^{\prime \prime \prime}+\left(\alpha_{l}-v\right) U_{l}^{\prime}+\left(4 \kappa^{3} b_{l}-3 \kappa^{2} a_{l}\right) U_{\tilde{l}}^{\prime}=0 .
$$

Here the following notation is used: ' $=\frac{d}{d \xi}, "=\frac{d^{2}}{d \xi^{2}},{ }^{(i v)}=\frac{d^{4}}{d \xi^{4}}, l=1,2$, and $\tilde{l}=3-l$. Eqs. and (5) reduce to the ODE

$$
b_{l} U_{l}^{(i v)}+6 \kappa^{2} b_{l} U_{l}^{\prime \prime}+\left(\kappa \alpha_{l}-\omega+\beta_{l}-3 \kappa^{4} b_{l}\right) U_{l}+\left(c_{l}+d_{l}\right) U_{l}^{3}=0,
$$

with the soliton velocity

and the constraints

$$
v=\alpha_{l}-8 \kappa^{3} b_{l}
$$

$$
\begin{aligned}
& U_{\tilde{l}}=U_{l}, \\
& a_{l}=4 \kappa b_{l} .
\end{aligned}
$$

Eq. (6) holds a formal solution

$$
U_{l}(\xi)=\sum_{i=1}^{N} \cos ^{i-1}\left(V_{l}(\xi)\right)\left[B_{i} \sin \left(V_{l}(\xi)\right)+A_{i} \cos \left(V_{l}(\xi)\right]+A_{0},\right.
$$

along with the ODE 


$$
V_{l}^{\prime}(\xi)=\sin \left(V_{l}(\xi)\right)
$$

and the exact solutions

$$
\begin{aligned}
& \sin \left(V_{l}(\xi)\right)=\operatorname{sech}(\xi), \quad \sin \left(V_{l}(\xi)\right)=i \operatorname{csch}(\xi), \\
& \cos \left(V_{l}(\xi)\right)=\tanh (\xi), \quad \cos \left(V_{l}(\xi)\right)=\operatorname{coth}(\xi),
\end{aligned}
$$

where $V_{l}(\xi)$ is a new positive function of $\xi$. The balance number is given by the integer $N$, and $A_{i}$ and $B_{i}$ are constants. Balancing $U_{l}^{(i v)}$ with $U_{l}^{3}$ in Eq. (6) gives $N=2$. Thus, Eq. (10) yields the solution

$$
U_{l}(\xi)=A_{0}+B_{1} \sin \left(V_{l}(\xi)\right)+A_{1} \cos \left(V_{l}(\xi)\right)+\cos \left(V_{l}(\xi)\right)\left(B_{2} \sin \left(V_{l}(\xi)\right)+A_{2} \cos \left(V_{l}(\xi)\right)\right) .
$$

Inserting Eqs. (13) and (11) into Eq. (6) gives rise to the following results:

$$
\begin{aligned}
& \kappa= \pm \frac{\sqrt{15}}{3}, A_{0}=0, A_{1}=0, A_{2}=0, B_{1}=0, \\
& B_{2}= \pm 2 \sqrt{\frac{30 b_{l}}{c_{l}+d_{l}}}, \alpha_{l}= \pm \frac{\sqrt{15}\left(8 b_{l}+3 \beta_{l}-3 \omega\right)}{15} .
\end{aligned}
$$

Substituting Eqs. (14) and (12) into Eq. (13) yields the combo dark-bright solitons

$$
\begin{aligned}
& q(x, t)= \pm 2 \sqrt{\frac{30 b_{1}}{c_{1}+d_{1}}} \tanh \left(x-\left(\alpha_{1}-8 \kappa^{3} b_{1}\right) t\right) \operatorname{sech}\left(x-\left(\alpha_{1}-8 \kappa^{3} b_{1}\right) t\right) e^{i\left(-\kappa x+\omega t+\theta_{0}\right)}, \\
& r(x, t)= \pm 2 \sqrt{\frac{30 b_{2}}{c_{2}+d_{2}}} \tanh \left(x-\left(\alpha_{2}-8 \kappa^{3} b_{2}\right) t\right) \operatorname{sech}\left(x-\left(\alpha_{2}-8 \kappa^{3} b_{2}\right) t\right) e^{i\left(-\kappa x+\omega t+\theta_{0}\right)}
\end{aligned}
$$

with

$$
b_{l}\left(c_{l}+d_{l}\right)>0
$$

and the combo singular solitons

$$
\begin{aligned}
& q(x, t)= \pm 2 \sqrt{-\frac{30 b_{1}}{c_{1}+d_{1}}} \operatorname{coth}\left(x-\left(\alpha_{1}-8 \kappa^{3} b_{1}\right) t\right) \operatorname{csch}\left(x-\left(\alpha_{1}-8 \kappa^{3} b_{1}\right) t\right) e^{i\left(-\kappa x+\omega t+\theta_{0}\right)} \\
& r(x, t)= \pm 2 \sqrt{-\frac{30 b_{2}}{c_{2}+d_{2}}} \operatorname{coth}\left(x-\left(\alpha_{2}-8 \kappa^{3} b_{2}\right) t\right) \operatorname{csch}\left(x-\left(\alpha_{2}-8 \kappa^{3} b_{2}\right) t\right) e^{i\left(-\kappa x+\omega t+\theta_{0}\right)}
\end{aligned}
$$

with

$$
b_{l}\left(c_{l}+d_{l}\right)<0
$$

\subsection{Parabolic law}

The governing model with the parabolic nonlinear refractive index can be written as

$$
\begin{aligned}
& i q_{t}+i a_{1} r_{x x x}+b_{1} r_{x x x x}+\left(c_{1}|q|^{2}+d_{1}|r|^{2}\right) q+\left(\lambda_{1}|q|^{4}+\mu_{1}|q|^{2}|r|^{2}+\zeta_{1}|r|^{4}\right) q+i \alpha_{1} q_{x}+\beta_{1} r=0, \\
& i r_{t}+i a_{2} q_{x x x}+b_{2} q_{x x x x}+\left(c_{2}|r|^{2}+d_{2}|q|^{2}\right) r+\left(\lambda_{2}|r|^{4}+\mu_{2}|r|^{2}|q|^{2}+\zeta_{2}|q|^{4}\right) r+i \alpha_{2} r_{x}+\beta_{2} q=0,
\end{aligned}
$$

where $c_{l}$ and $\lambda_{l}$ are the coefficients of SPM, while $d_{l}, \mu_{l}$ and $\zeta_{l}$ represent the coefficients of XPM. Substituting Eq. (3) into Eqs. (19) and (20) yields the real part

$$
\begin{aligned}
& b_{l} U_{\tilde{l}}^{(i v)}+\left(3 \kappa a_{l}-6 \kappa^{2} b_{l}\right) U_{\tilde{l}}^{\prime \prime}+\left(\kappa \alpha_{l}-\omega\right) U_{l}+\left(\beta_{l}-\kappa^{3} a_{l}+\kappa^{4} b_{l}\right) U_{\tilde{l}} \\
& +c_{l} U_{l}^{3}+d_{l} U_{l} U_{\tilde{l}}^{2}+\lambda_{l} U_{l}^{5}+\mu_{l} U_{l}^{3} U_{\tilde{l}}^{2}+\zeta_{l} U_{l} U_{\tilde{l}}^{4}=0,
\end{aligned}
$$


and the imaginary part

$$
\left(a_{l}-4 \kappa b_{l}\right) U_{\tilde{l}}^{\prime \prime \prime}+\left(\alpha_{l}-v\right) U_{l}^{\prime}+\left(4 \kappa^{3} b_{l}-3 \kappa^{2} a_{l}\right) U_{\tilde{l}}^{\prime}=0 .
$$

Eqs. (21) and (22) reduce to the ODE

$$
b_{l} U_{l}^{(i v)}+6 \kappa^{2} b_{l} U_{l}^{\prime \prime}+\left(\kappa \alpha_{l}-\omega+\beta_{l}-3 \kappa^{4} b_{l}\right) U_{l}+\left(c_{l}+d_{l}\right) U_{l}^{3}+\left(\lambda_{l}+\mu_{l}+\zeta_{l}\right) U_{l}^{5}=0
$$

with the constraints

$$
\begin{aligned}
& U_{\tilde{l}}=U_{l} \\
& a_{l}=4 \kappa b_{l}, \\
& v=\alpha_{l}-8 \kappa^{3} b_{l} .
\end{aligned}
$$

Balancing $U_{l}^{(i v)}$ with $U_{l}^{5}$ in Eq. (23) gives $N=1$. Thus, Eq. (10) has the solution

$$
U_{l}(\xi)=B_{1} \sin \left(V_{l}(\xi)\right)+A_{1} \cos \left(V_{l}(\xi)\right)+A_{0} .
$$

Inserting Eqs. (27) and (11) into Eq. (23) gives rise to the following results:

Case 1:

$$
\begin{aligned}
A_{0}=0, B_{1}=0 \\
b_{l}=\frac{\kappa \alpha_{l}-\omega+\beta_{l}}{3 \kappa^{4}+12 \kappa^{2}-16}, \quad A_{1}= \pm 2 \sqrt{-\frac{3 \kappa^{3} \alpha_{l}-3 \kappa^{2} \omega+3 \kappa^{2} \beta_{l}-10 \kappa \alpha_{l}+10 \omega-10 \beta_{l}}{3 \kappa^{4} c_{l}+3 \kappa^{4} d_{l}+12 \kappa^{2} c_{l}+12 \kappa^{2} d_{l}-16 c_{l}-16 d_{l}}} \\
\zeta_{l}=-\frac{1}{2\left(3 \kappa^{2}-10\right)^{2}\left(\kappa \alpha_{l}-\omega+\beta_{l}\right)} \\
\times\left(18 \kappa^{5} \alpha_{l} \lambda_{l}+18 \kappa^{5} \alpha_{l} \mu_{l}-18 \kappa^{4} \omega \lambda_{l}-18 \kappa^{4} \omega \mu_{l}+18 \kappa^{4} \beta_{l} \lambda_{l}+18 \kappa^{4} \beta_{l} \mu_{l}+9 \kappa^{4} c_{l}^{2}\right. \\
+18 \kappa^{4} c_{l} d_{l}+9 \kappa^{4} d_{l}^{2}-120 \kappa^{3} \alpha_{l} \lambda_{l}-120 \kappa^{3} \alpha_{l} \mu_{l}+120 \kappa^{2} \omega \lambda_{l}+120 \kappa^{2} \omega \mu_{l}-120 \kappa^{2} \beta_{l} \lambda_{l} \\
-120 \kappa^{2} \beta_{l} \mu_{l}+36 \kappa^{2} c_{l}^{2}+72 \kappa^{2} c_{l} d_{l}+36 \kappa^{2} d_{l}^{2}+200 \kappa \alpha_{l} \lambda_{l}+200 \kappa \alpha_{l} \mu_{l} \\
\left.-200 \omega \lambda_{l}-200 \omega \mu_{l}+200 \beta_{l} \lambda_{l}-48 c_{l}^{2}+200 \beta_{l} \mu_{l}-96 c_{l} d_{l}-48 d_{l}^{2}\right) .
\end{aligned}
$$

Substituting Eqs. (28) and (12) into Eq. (27) yields the dark solitons

$$
\begin{aligned}
q(x, t) & = \pm 2 \sqrt{-\frac{3 \kappa^{3} \alpha_{1}-3 \kappa^{2} \omega+3 \kappa^{2} \beta_{1}-10 \kappa \alpha_{1}+10 \omega-10 \beta_{1}}{3 \kappa^{4} c_{1}+3 \kappa^{4} d_{1}+12 \kappa^{2} c_{1}+12 \kappa^{2} d_{1}-16 c_{1}-16 d_{1}}} \\
& \times \tanh \left(x-\left(\alpha_{1}-8 \kappa^{3} b_{1}\right) t\right) e^{i\left(-\kappa x+\omega t+\theta_{0}\right)} \\
r(x, t) & = \pm 2 \sqrt{-\frac{3 \kappa^{3} \alpha_{2}-3 \kappa^{2} \omega+3 \kappa^{2} \beta_{2}-10 \kappa \alpha_{2}+10 \omega-10 \beta_{2}}{3 \kappa^{4} c_{2}+3 \kappa^{4} d_{2}+12 \kappa^{2} c_{2}+12 \kappa^{2} d_{2}-16 c_{2}-16 d_{2}}} \\
& \times \tanh \left(x-\left(\alpha_{2}-8 \kappa^{3} b_{2}\right) t\right) e^{i\left(-\kappa x+\omega t+\theta_{0}\right)}
\end{aligned}
$$

and the singular solitons

$$
\begin{aligned}
q(x, t) & = \pm 2 \sqrt{-\frac{3 \kappa^{3} \alpha_{1}-3 \kappa^{2} \omega+3 \kappa^{2} \beta_{1}-10 \kappa \alpha_{1}+10 \omega-10 \beta_{1}}{3 \kappa^{4} c_{1}+3 \kappa^{4} d_{1}+12 \kappa^{2} c_{1}+12 \kappa^{2} d_{1}-16 c_{1}-16 d_{1}}} \\
& \times \operatorname{coth}\left(x-\left(\alpha_{1}-8 \kappa^{3} b_{1}\right) t\right) e^{i\left(-\kappa x+\omega t+\theta_{0}\right)}
\end{aligned}
$$




$$
\begin{aligned}
r(x, t) & = \pm 2 \sqrt{-\frac{3 \kappa^{3} \alpha_{2}-3 \kappa^{2} \omega+3 \kappa^{2} \beta_{2}-10 \kappa \alpha_{2}+10 \omega-10 \beta_{2}}{3 \kappa^{4} c_{2}+3 \kappa^{4} d_{2}+12 \kappa^{2} c_{2}+12 \kappa^{2} d_{2}-16 c_{2}-16 d_{2}}} \\
& \times \operatorname{coth}\left(x-\left(\alpha_{2}-8 \kappa^{3} b_{2}\right) t\right) e^{i\left(-\kappa x+\omega t+\theta_{0}\right)},
\end{aligned}
$$

with

$$
\begin{aligned}
& \left(3 \kappa^{3} \alpha_{l}-3 \kappa^{2} \omega+3 \kappa^{2} \beta_{l}-10 \kappa \alpha_{l}+10 \omega-10 \beta_{l}\right) \\
& \times\left(3 \kappa^{4} c_{l}+3 \kappa^{4} d_{l}+12 \kappa^{2} c_{l}+12 \kappa^{2} d_{l}-16 c_{l}-16 d_{l}\right)<0 .
\end{aligned}
$$

Case 2:

$$
\begin{aligned}
& A_{0}=0, A_{1}=0, b_{l}=\frac{\kappa \alpha_{l}-\omega+\beta_{l}}{3 \kappa^{4}-6 \kappa^{2}-1}, \quad B_{1}= \pm 2 \sqrt{\frac{3 \kappa^{3} \alpha_{l}-3 \kappa^{2} \omega+3 \kappa^{2} \beta_{l}+5 \kappa \alpha_{l}-5 \omega+5 \beta_{l}}{3 \kappa^{4} c_{l}+3 \kappa^{4} d_{l}-6 \kappa^{2} c_{l}-6 \kappa^{2} d_{l}-c_{l}-d_{l}}} \\
& \zeta_{l}=-\frac{1}{2\left(3 \kappa^{2}+5\right)^{2}\left(\kappa \alpha_{l}-\omega+\beta_{l}\right)}\left(18 \kappa^{5} \alpha_{l} \lambda_{l}+18 \kappa^{5} \alpha_{l} \mu_{l}-18 \kappa^{4} \omega \lambda_{l}-18 \kappa^{4} \omega \mu_{l}\right. \\
& +18 \kappa^{4} \beta_{l} \lambda_{l}+18 \kappa^{4} \beta_{l} \mu_{l}+9 \kappa^{4} c_{l}^{2}+18 \kappa^{4} c_{l} d_{l}+9 \kappa^{4} d_{l}^{2}+60 \kappa^{3} \alpha_{l} \lambda_{l}+60 \kappa^{3} \alpha_{l} \mu_{l} \\
& -60 \kappa^{2} \omega \lambda_{l}-60 \kappa^{2} \omega \mu_{l}+60 \kappa^{2} \beta_{l} \lambda_{l}+60 \kappa^{2} \beta_{l} \mu_{l}-18 \kappa^{2} c_{l}^{2}-36 \kappa^{2} c_{l} d_{l}-18 \kappa^{2} d_{l}^{2} \\
& \left.+50 \kappa \alpha_{l} \lambda_{l}+50 \kappa \alpha_{l} \mu_{l}-50 \omega \lambda_{l}-50 \omega \mu_{l}+50 \beta_{l} \lambda_{l}+50 \beta_{l} \mu_{l}-3 c_{l}^{2}-6 c_{l} d_{l}-3 d_{l}^{2}\right) .
\end{aligned}
$$

Inserting Eqs. (33) and (12) into Eq. (27) yields the bright solitons

$$
\begin{aligned}
q(x, t) & = \pm 2 \sqrt{\frac{3 \kappa^{3} \alpha_{1}-3 \kappa^{2} \omega+3 \kappa^{2} \beta_{1}+5 \kappa \alpha_{1}-5 \omega+5 \beta_{1}}{3 \kappa^{4} c_{1}+3 \kappa^{4} d_{1}-6 \kappa^{2} c_{1}-6 \kappa^{2} d_{1}-c_{1}-d_{1}}} \\
& \times \operatorname{sech}\left(x-\left(\alpha_{1}-8 \kappa^{3} b_{1}\right) t\right) e^{i\left(-\kappa x+\omega t+\theta_{0}\right)} \\
r(x, t) & = \pm 2 \sqrt{\frac{3 \kappa^{3} \alpha_{2}-3 \kappa^{2} \omega+3 \kappa^{2} \beta_{2}+5 \kappa \alpha_{2}-5 \omega+5 \beta_{2}}{3 \kappa^{4} c_{2}+3 \kappa^{4} d_{2}-6 \kappa^{2} c_{2}-6 \kappa^{2} d_{2}-c_{2}-d_{2}}} \\
& \times \operatorname{sech}\left(x-\left(\alpha_{2}-8 \kappa^{3} b_{2}\right) t\right) e^{i\left(-\kappa x+\omega t+\theta_{0}\right)},
\end{aligned}
$$

with

$$
\left(3 \kappa^{3} \alpha_{l}-3 \kappa^{2} \omega+3 \kappa^{2} \beta_{l}+5 \kappa \alpha_{l}-5 \omega+5 \beta_{l}\right) \times\left(3 \kappa^{4} c_{l}+3 \kappa^{4} d_{l}-6 \kappa^{2} c_{l}-6 \kappa^{2} d_{l}-c_{l}-d_{l}\right)>0,
$$

and the singular solitons

$$
\begin{aligned}
r(x, t) & = \pm 2 \sqrt{\frac{3 \kappa^{3} \alpha_{1}-3 \kappa^{2} \omega+3 \kappa^{2} \beta_{1}+5 \kappa \alpha_{1}-5 \omega+5 \beta_{1}}{3 \kappa^{4} c_{1}+3 \kappa^{4} d_{1}-6 \kappa^{2} c_{1}-6 \kappa^{2} d_{1}-c_{1}-d_{1}}} \\
& \times \operatorname{csch}\left(x-\left(\alpha_{2}-8 \kappa^{3} b_{2}\right) t\right) e^{i\left(-\kappa x+\omega t+\theta_{0}\right)}, \\
r(x, t) & = \pm 2 \sqrt{-\frac{3 \kappa^{3} \alpha_{2}-3 \kappa^{2} \omega+3 \kappa^{2} \beta_{2}+5 \kappa \alpha_{2}-5 \omega+5 \beta_{2}}{3 \kappa^{4} c_{2}+3 \kappa^{4} d_{2}-6 \kappa^{2} c_{2}-6 \kappa^{2} d_{2}-c_{2}-d_{2}}} \\
& \times \operatorname{csch}\left(x-\left(\alpha_{2}-8 \kappa^{3} b_{2}\right) t\right) e^{i\left(-\kappa x+\omega t+\theta_{0}\right)},
\end{aligned}
$$

with

$$
\left(3 \kappa^{3} \alpha_{l}-3 \kappa^{2} \omega+3 \kappa^{2} \beta_{l}+5 \kappa \alpha_{l}-5 \omega+5 \beta_{l}\right) \times\left(3 \kappa^{4} c_{l}+3 \kappa^{4} d_{l}-6 \kappa^{2} c_{l}-6 \kappa^{2} d_{l}-c_{l}-d\right)_{l}<0 .
$$


Case 3:

$$
\begin{aligned}
A_{0}= & 0, b_{l}=\frac{\kappa \alpha_{l}-\omega+\beta_{l}}{3 \kappa^{4}+3 \kappa^{2}-1}, \quad A_{1}= \pm \sqrt{-\frac{6 \kappa^{3} \alpha_{l}-6 \kappa^{2} \omega+6 \kappa^{2} \beta_{l}-5 \kappa \alpha_{l}+5 \omega-5 \beta_{l}}{6 \kappa^{4} c_{l}+6 \kappa^{4} d_{l}+6 \kappa^{2} c_{l}+6 \kappa^{2} d_{l}-2 c_{l}-2 d_{l}}}, \\
B_{1}= & \sqrt{\frac{6 \kappa^{3} \alpha_{l}-6 \kappa^{2} \omega+6 \kappa^{2} \beta_{l}-5 \kappa \alpha_{l}+5 \omega-5 \beta_{l}}{6 \kappa^{4} c_{l}+6 \kappa^{4} d_{l}+6 \kappa^{2} c_{l}+6 \kappa^{2} d_{l}-2 c_{l}-2 d_{l}}}, \\
\zeta_{l}= & -\frac{1}{\left(6 \kappa^{2}-5\right)^{2}\left(\kappa \alpha_{l}-\omega+\beta_{l}\right)}\left(36 \alpha^{5} \alpha_{l} \lambda_{l}+36 \kappa^{5} \alpha_{l} \mu_{l}-36 \kappa^{4} \omega \lambda_{l}-36 \kappa^{4} \omega \mu_{l}\right. \\
& +36 \kappa^{4} \beta_{l} \lambda_{l}+36 \kappa^{4} \beta_{l} \mu_{l}+18 \kappa^{4} c_{l}^{2}+36 \kappa^{4} c_{l} d_{l}+18 \kappa^{4} d_{l}^{2}-60 \kappa^{3} \alpha_{l} \lambda_{l}-60 \kappa^{3} \alpha_{l} \mu_{l} \\
& +60 \kappa^{2} \omega \lambda_{l}+60 \kappa^{2} \omega \mu_{l}-60 \kappa^{2} \beta_{l} \lambda_{l}-60 \kappa^{2} \beta_{l} \mu_{l}+18 \kappa^{2} c_{l}^{2}+36 \kappa^{2} c_{l} d_{l}+18 \kappa^{2} d_{l}^{2} \\
& \left.+25 \kappa \alpha_{l} \lambda_{l}+25 \kappa \alpha_{l} \mu_{l}-25 \omega \lambda_{l}-25 \omega \mu_{l}+25 \beta_{l} \lambda_{l}+25 \beta_{l} \mu_{l}-6 c_{l}^{2}-12 c_{l} d_{l}-6 d_{l}^{2}\right) .
\end{aligned}
$$

Substituting Eqs. (38) and (12) into Eq. (27) causes the combo singular solitons

$$
\begin{aligned}
q(x, t) & = \pm \sqrt{-\frac{6 \kappa^{3} \alpha_{1}-6 \kappa^{2} \omega+6 \kappa^{2} \beta_{1}-5 \kappa \alpha_{1}+5 \omega-5 \beta_{1}}{6 \kappa^{4} c_{1}+6 \kappa^{4} d_{1}+6 \kappa^{2} c_{1}+6 \kappa^{2} d_{1}-2 c_{1}-2 d_{1}}} \\
& \times\left(\operatorname{coth}\left(x-\left(\alpha_{1}-8 \kappa^{3} b_{1}\right) t\right)+\operatorname{csch}\left(x-\left(\alpha_{1}-8 \kappa^{3} b_{1}\right) t\right)\right) e^{i\left(-\kappa x+\omega t+\theta_{0}\right)}, \\
r(x, t) & = \pm \sqrt{-\frac{6 \kappa^{3} \alpha_{2}-6 \kappa^{2} \omega+6 \kappa^{2} \beta_{2}-5 \kappa \alpha_{2}+5 \omega-5 \beta_{2}}{6 \kappa^{4} c_{2}+6 \kappa^{4} d_{2}+6 \kappa^{2} c_{2}+6 \kappa^{2} d_{2}-2 c_{2}-2 d_{2}}} \\
& \times\left(\operatorname{coth}\left(x-\left(\alpha_{2}-8 \kappa^{3} b_{2}\right) t\right)+\operatorname{csch}\left(x-\left(\alpha_{2}-8 \kappa^{3} b_{2}\right) t\right)\right) e^{i\left(-\kappa x+\omega t+\theta_{0}\right)},
\end{aligned}
$$

with

$$
\begin{aligned}
& \left(-6 \kappa^{3} \alpha_{l}+6 \kappa^{2} \omega-6 \kappa^{2} \beta_{l}+5 \kappa \alpha_{l}-5 \omega+5 \beta_{l}\right) \\
& \times\left(6 \kappa^{4} c_{l}+6 \kappa^{4} d_{l}+6 \kappa^{2} c_{l}+6 \kappa^{2} d_{l}-2 c_{l}-2 d\right)_{l}<0 .
\end{aligned}
$$

Case 4:

$$
\begin{aligned}
& \kappa= \pm \frac{\sqrt{30}}{6}, \quad A_{0}=0, \quad A_{1}= \pm \sqrt{-\frac{15 b_{l}}{c_{l}+d_{l}}}, \quad B_{1}= \pm \sqrt{-\frac{15 b_{l}}{c_{l}+d_{l}}} \\
& \alpha_{l}= \pm \frac{\sqrt{30}\left(12 \omega+133 b_{l}-12 \beta_{l}\right)}{60} \\
& \zeta_{l}=-\frac{75 b_{l} \lambda_{l}+75 b_{l} \mu_{l}-2 c_{l}^{2}-4 c_{l} d_{l}-2 d_{l}^{2}}{75 b_{l}} .
\end{aligned}
$$

Inserting Eqs. (41) and (12) into Eq. (27) produces the combo dark-bright solitons

$$
\begin{aligned}
& q(x, t)= \pm \sqrt{-\frac{15 b_{1}}{c_{1}+d_{1}}}\left(\tanh \left(x-\left(\alpha_{1}-8 \kappa^{3} b_{1}\right) t\right)+\operatorname{sech}\left(x-\left(\alpha_{1}-8 \kappa^{3} b_{1}\right) t\right)\right) e^{i\left(-\kappa x+\omega t+\theta_{0}\right)} \\
& r(x, t)= \pm \sqrt{-\frac{15 b_{2}}{c_{2}+d_{2}}}\left(\tanh \left(x-\left(\alpha_{2}-8 \kappa^{3} b_{2}\right) t\right)+\operatorname{sech}\left(x-\left(\alpha_{2}-8 \kappa^{3} b_{2}\right) t\right)\right) e^{i\left(-\kappa x+\omega t+\theta_{0}\right)}
\end{aligned}
$$

with

$$
b_{l}\left(c_{l}+d_{l}\right)<0
$$




\subsection{Quadratic-cubic law}

The governing model with the quadratic-cubic nonlinearity of the refractive index is structured as

$$
\begin{aligned}
& i q_{t}+i a_{1} r_{x x x}+b_{1} r_{x x x x}+c_{1} q \sqrt{|q|^{2}+|r|^{2}+q r^{*}+q^{*} r}+\left(d_{1}|q|^{2}+e_{1}|r|^{2}\right) q+\lambda_{1} r^{2} q^{*}+i \alpha_{1} q_{x}+\beta_{1} r=0 \\
& i r_{t}+i a_{2} q_{x x x}+b_{2} q_{x x x x}+c_{2} r \sqrt{|r|^{2}+|q|^{2}+q r^{*}+q^{*} r}+\left(d_{2}|r|^{2}+e_{2}|q|^{2}\right) r+\lambda_{2} q^{2} r^{*}+i \alpha_{2} r_{x}+\beta_{2} q=0
\end{aligned}
$$

where $d_{l}$ and $e_{l}$ are the coefficients of respectively SPM and XPM, and $\lambda_{l}$ stand for the coefficients of four-wave mixing. In the case of quadratic nonlinearity, $c_{l}$ represent the coefficients of SPM and XPM, along with four-wave mixing.

Substituting Eq. (3) into Eqs. (44) and (45) leads to the real part

$$
\begin{aligned}
& b_{l} U_{\tilde{l}}^{(i v)}+\left(3 \kappa a_{l}-6 \kappa^{2} b_{l}\right) U_{\tilde{l}}^{\prime \prime}+\left(\kappa \alpha_{l}-\omega\right) U_{l} \\
& +\left(\kappa^{4} b_{l}-\kappa^{3} a_{l}+\beta_{l}\right) U_{\tilde{l}}+c_{l} U_{l}^{2}+c_{l} U_{l} U_{\tilde{l}}+d_{l} U_{l}^{3}+\left(e_{l}+\lambda_{l}\right) U_{l} U_{\tilde{l}}^{2}=0,
\end{aligned}
$$

and the imaginary part

$$
\left(a_{l}-4 \kappa b_{l}\right) U_{\tilde{l}}^{\prime \prime \prime}+\left(\alpha_{l}-v\right) U_{l}^{\prime}+\left(4 \kappa^{3} b_{l}-3 \kappa^{2} a_{l}\right) U_{\tilde{l}}^{\prime}=0 .
$$

Eqs. (46) and (47) reduce to the ODE

$$
b_{l} U_{l}^{(i v)}+6 \kappa^{2} b_{l} U_{l}^{\prime \prime}+\left(\kappa \alpha_{l}-\omega+\beta_{l}-3 \kappa^{4} b_{l}\right) U_{l}+2 c_{l} U_{l}^{2}+\left(d_{l}+e_{l}+\lambda_{l}\right) U_{l}^{3}=0,
$$

with the constraints

$$
\begin{aligned}
& U_{\tilde{l}}=U_{l}, \\
& a_{l}=4 \kappa b_{l}, \\
& v=\alpha_{l}-8 \kappa^{3} b_{l} .
\end{aligned}
$$

Here Eq. (48) admits Eq. (13). Inserting Eqs. (13) and (11) into Eq. (48) gives rise to the following results:

Case 1:

$$
\begin{aligned}
& A_{0}= \pm 2 \sqrt{-\frac{30 b_{l}}{d_{l}+e_{l}+\lambda_{l}}}, \quad A_{1}=0, \quad A_{2}= \pm 2 \sqrt{-\frac{30 b_{l}}{d_{l}+e_{l}+\lambda_{l}}}, \\
& B_{1}=0, \quad B_{2}=0, \quad \beta_{l}=3 \kappa^{4} b_{l}-24 \kappa^{2} b_{l}-\kappa \alpha_{l}+\omega-16 b_{l}, \\
& c_{l}= \pm \frac{\left(3 \kappa^{2}+10\right) \sqrt{-30 b_{l}\left(d_{l}+e_{l}+\lambda_{l}\right)}}{10} .
\end{aligned}
$$

Substituting Eqs. (52) and (12) into Eq. (13) yields the dark solitons

$$
\begin{aligned}
& q(x, t)= \pm 2 \sqrt{-\frac{30 b_{1}}{d_{1}+e_{1}+\lambda_{1}}}\left\{1+\tanh ^{2}\left(x-\left(\alpha_{1}-8 \kappa^{3} b_{1}\right) t\right)\right\} e^{i\left(-\kappa x+\omega t+\theta_{0}\right)}, \\
& r(x, t)= \pm 2 \sqrt{-\frac{30 b_{2}}{d_{2}+e_{2}+\lambda_{2}}}\left\{1+\tanh ^{2}\left(x-\left(\alpha_{2}-8 \kappa^{3} b_{2}\right) t\right)\right\} e^{i\left(-\kappa x+\omega t+\theta_{0}\right)},
\end{aligned}
$$

and the singular solitons

$$
q(x, t)= \pm 2 \sqrt{-\frac{30 b_{1}}{d_{1}+e_{1}+\lambda_{1}}}\left\{1+\operatorname{coth}^{2}\left(x-\left(\alpha_{1}-8 \kappa^{3} b_{1}\right) t\right)\right\} e^{i\left(-\kappa x+\omega t+\theta_{0}\right)},
$$




$$
r(x, t)= \pm 2 \sqrt{-\frac{30 b_{2}}{d_{2}+e_{2}+\lambda_{2}}}\left\{1+\operatorname{coth}^{2}\left(x-\left(\alpha_{2}-8 \kappa^{3} b_{2}\right) t\right)\right\} e^{i\left(-\kappa x+\omega t+\theta_{0}\right)}
$$

with

$$
b_{l}\left(d_{l}+e_{l}+\lambda_{l}\right)<0 .
$$

Case 2:

$$
\begin{aligned}
& A_{0}= \pm \sqrt{\frac{11 b_{l}}{d_{l}+e_{l}+\lambda_{l}}}, \quad A_{1}=0, \quad A_{2}=0, \quad B_{1}=0, \quad B_{2}= \pm 2 \sqrt{\frac{30 b_{l}}{d_{l}+e_{l}+\lambda_{l}}}, \\
& \kappa= \pm \frac{\sqrt{15}}{3}, \quad \alpha_{l}= \pm \frac{\sqrt{15}\left(3 \omega+91 b_{l}-3 \beta_{l}\right)}{15}, \quad c_{l}= \pm \frac{3}{2} \sqrt{11 b_{l}\left(d_{l}+e_{l}+\lambda_{l}\right)} .
\end{aligned}
$$

Inserting Eqs. (57) and (12) into Eq. (13) yields the combo dark-bright solitons

$$
\begin{aligned}
q(x, t) & = \pm \sqrt{\frac{b_{1}}{d_{1}+e_{1}+\lambda_{1}}}\left\{\sqrt{11}+2 \sqrt{30} \tanh \left(x-\left(\alpha_{1}-8 \kappa^{3} b_{1}\right) t\right) \operatorname{sech}\left(x-\left(\alpha_{1}-8 \kappa^{3} b_{1}\right) t\right)\right\} \\
& \times e^{i\left(-\kappa x+\omega t+\theta_{0}\right)}, \\
r(x, t) & = \pm \sqrt{\frac{b_{2}}{d_{2}+e_{2}+\lambda_{2}}}\left\{\sqrt{11} \pm 2 \sqrt{30} \tanh \left(x-\left(\alpha_{2}-8 \kappa^{3} b_{2}\right) t\right) \operatorname{sech}\left(x-\left(\alpha_{2}-8 \kappa^{3} b_{2}\right) t\right)\right\} \\
& \times e^{i\left(-\kappa x+\omega t+\theta_{0}\right)},
\end{aligned}
$$

with the constraint

$$
b_{l}\left(d_{l}+e_{l}+\lambda_{l}\right)>0
$$

Case 3:

$$
\begin{aligned}
& A_{0}= \pm \sqrt{-\frac{30 b_{l}}{d_{l}+e_{l}+\lambda_{l}}}, A_{2}= \pm \sqrt{-\frac{30 b_{l}}{d_{l}+e_{l}+\lambda_{l}}}, A_{1}=0, \\
& B_{1}=0, B_{2}= \pm \sqrt{\frac{30 b_{l}}{d_{l}+e_{l}+\lambda_{l}}}, c_{l}= \pm \frac{6 \kappa^{2}+5}{2} \sqrt{-\frac{3 b_{l}\left(d_{l}+e_{l}+\lambda_{l}\right)}{10}}, \\
& \beta_{l}=3 \kappa^{4} b_{l}-6 \kappa^{2} b_{l}-\kappa \alpha_{l}+\omega-b_{l} .
\end{aligned}
$$

Substituting Eqs. (60) and (12) into Eq. (13) reveals the combo singular solitons

$$
\begin{aligned}
q(x, t) & = \pm \sqrt{-\frac{30 b_{1}}{d_{1}+e_{1}+\lambda_{1}}} \\
& \times\left\{1+\operatorname{coth}^{2}\left(x-\left(\alpha_{1}-8 \kappa^{3} b_{1}\right) t\right)+\operatorname{coth}\left(x-\left(\alpha_{1}-8 \kappa^{3} b_{1}\right) t\right) \operatorname{csch}\left(x-\left(\alpha_{1}-8 \kappa^{3} b_{1}\right) t\right)\right\} \\
& \times e^{i\left(-\kappa x+\omega t+\theta_{0}\right)}, \\
r(x, t)= & \pm \sqrt{-\frac{30 b_{2}}{d_{2}+e_{2}+\lambda_{2}}} \\
& \times\left\{1+\operatorname{coth}^{2}\left(x-\left(\alpha_{2}-8 \kappa^{3} b_{2}\right) t\right)+\operatorname{coth}\left(x-\left(\alpha_{2}-8 \kappa^{3} b_{2}\right) t\right) \operatorname{csch}\left(x-\left(\alpha_{2}-8 \kappa^{3} b_{2}\right) t\right)\right\} \\
& \times e^{i\left(-\kappa x+\omega t+\theta_{0}\right)},
\end{aligned}
$$

with

$$
b_{l}\left(d_{l}+e_{l}+\lambda_{l}\right)<0 \text {. }
$$


Case 4:

$$
\begin{aligned}
& A_{0}=0, A_{1}=0, A_{2}= \pm 2 \sqrt{-\frac{30 b_{l}}{d_{l}+e_{l}+\lambda_{l}}}, B_{2}=0, B_{1}=0, \\
& \beta_{l}=\frac{2 \sqrt{3} \alpha_{l}}{3}+\omega-\frac{200 b_{l}}{3}, \kappa= \pm \frac{2 \sqrt{3}}{3}, c_{l}= \pm \sqrt{-\frac{384 b_{l}\left(d_{l}+e_{l}+\lambda_{l}\right)}{5}} .
\end{aligned}
$$

Inserting Eqs. (63) and (12) into Eq. (13) yields the dark solitons

$$
\begin{aligned}
& q(x, t)= \pm 2 \sqrt{-\frac{30 b_{1}}{d_{1}+e_{1}+\lambda_{1}}} \tanh ^{2}\left(x-\left(\alpha_{1}-8 \kappa^{3} b_{1}\right) t\right) e^{i\left(-\kappa x+\omega t+\theta_{0}\right)}, \\
& r(x, t)= \pm 2 \sqrt{-\frac{30 b_{2}}{d_{2}+e_{2}+\lambda_{2}}} \tanh ^{2}\left(x-\left(\alpha_{2}-8 \kappa^{3} b_{2}\right) t\right) e^{i\left(-\kappa x+\omega t+\theta_{0}\right)},
\end{aligned}
$$

and the singular solitons

$$
\begin{aligned}
q(x, t) & = \pm 2 \sqrt{-\frac{30 b_{1}}{d_{1}+e_{1}+\lambda_{1}}} \operatorname{coth}^{2}\left(x-\left(\alpha_{1}-8 \kappa^{3} b_{1}\right) t\right) \\
& \times e^{i\left(-\kappa x+\omega t+\theta_{0}\right)}, \\
r(x, t) & = \pm 2 \sqrt{-\frac{30 b_{2}}{d_{2}+e_{2}+\lambda_{2}}} \operatorname{coth}^{2}\left(x-\left(\alpha_{2}-8 \kappa^{3} b_{2}\right) t\right) \\
& \times e^{i\left(-\kappa x+\omega t+\theta_{0}\right)},
\end{aligned}
$$

with

$$
b_{l}\left(d_{l}+e_{l}+\lambda_{l}\right)<0 \text {. }
$$

Case 5:

$$
\begin{aligned}
& A_{0}=0, A_{1}=0, A_{2}= \pm \sqrt{-\frac{30 b_{l}}{d_{l}+e_{l}+\lambda_{l}}}, B_{1}=0, B_{2}= \pm \sqrt{\frac{30 b_{l}}{d_{l}+e_{l}+\lambda_{l}}} \\
& \kappa= \pm \frac{2 \sqrt{3}}{3}, \beta_{l}= \pm \frac{2 \sqrt{3} \alpha_{l}}{3}+\omega-\frac{47 b_{l}}{3}, c_{l}= \pm \sqrt{-\frac{867 b_{l}\left(d_{l}+e_{l}+\lambda_{l}\right)}{40}} .
\end{aligned}
$$

Substituting Eqs. (68) and (12) into Eq. (13) produces the combo singular solitons

$$
\begin{aligned}
q(x, t) & = \pm \sqrt{-\frac{30 b_{1}}{d_{1}+e_{1}+\lambda_{1}}} \\
& \times\left(\operatorname{coth}^{2}\left(x-\left(\alpha_{1}-8 \kappa^{3} b_{1}\right) t\right)+\operatorname{coth}\left(x-\left(\alpha_{1}-8 \kappa^{3} b_{1}\right) t\right) \operatorname{csch}\left(x-\left(\alpha_{1}-8 \kappa^{3} b_{1}\right) t\right)\right) \\
& \times e^{i\left(-\kappa x+\omega t+\theta_{0}\right)}, \\
r(x, t)= & \pm \sqrt{-\frac{30 b_{2}}{d_{2}+e_{2}+\lambda_{2}}} \\
& \times\left\{\operatorname{coth}^{2}\left(x-\left(\alpha_{2}-8 \kappa^{3} b_{2}\right) t\right)+\operatorname{coth}\left(x-\left(\alpha_{2}-8 \kappa^{3} b_{2}\right) t\right) \operatorname{csch}\left(x-\left(\alpha_{2}-8 \kappa^{3} b_{2}\right) t\right)\right\} \\
& \times e^{i\left(-\kappa x+\omega t+\theta_{0}\right)},
\end{aligned}
$$

with $b_{l}\left(d_{l}+e_{l}+\lambda_{l}\right)<0$.

\subsection{Parabolic-nonlocal combo law}

The governing system with the parabolic-nonlocal combo nonlinear refractive index is given by 


$$
\begin{aligned}
& i q_{t}+i a_{1} r_{x x x}+b_{1} r_{x x x x}+\left(c_{1}|q|^{2}+d_{1}|r|^{2}\right)_{x x} q \\
& +\left(\lambda_{1}|q|^{4}+\mu_{1}|q|^{2}|r|^{2}+\zeta_{1}|r|^{4}\right) q+i \alpha_{1} q_{x}+\beta_{1} r=0, \\
& i r_{t}+i a_{2} q_{x x x}+b_{2} q_{x x x x}+\left(c_{2}|r|^{2}+d_{2}|q|^{2}\right)_{x x} r \\
& +\left(\lambda_{2}|r|^{4}+\mu_{2}|r|^{2}|q|^{2}+\zeta_{2}|q|^{4}\right) r+i \alpha_{2} r_{x}+\beta_{2} q=0,
\end{aligned}
$$

where $c_{l}$ and $\lambda_{l}$ are the coefficients of SPM, while $d_{l}, \mu_{l}$ and $\zeta_{l}$ represent the coefficients of XPM.

Substituting Eq. (3) into Eqs. (71) and (72) leads to the real part

$$
\begin{aligned}
& b_{l} U_{\tilde{l}}^{(i v)}+\left(3 \kappa a_{l}-6 \kappa^{2} b_{l}\right) U_{\tilde{l}}^{\prime \prime}+\left(\kappa \alpha_{l}-\omega\right) U_{l}+\left(\beta_{l}-\kappa^{3} a_{l}+\kappa^{4} b_{l}\right) U_{\tilde{l}}+2 c_{l} U_{l}\left(U_{l}^{\prime}\right)^{2} \\
& +2 d_{l} U_{l}\left(U_{\tilde{l}}^{\prime}\right)^{2}+2 c_{l} U_{l}^{2} U_{l}^{\prime \prime}+2 d_{l} U_{l} U_{\tilde{l}} U_{\tilde{l}}^{\prime \prime}+\lambda_{l} U_{l}^{5}+\mu_{l} U_{l}^{3} U_{\tilde{l}}^{2}+\zeta_{l} U_{l} U_{\tilde{l}}^{4}=0,
\end{aligned}
$$

and the imaginary part

$$
\left(a_{l}-4 \kappa b_{l}\right) U_{\tilde{l}}^{\prime \prime \prime}+\left(\alpha_{l}-v\right) U_{l}^{\prime}+\left(4 \kappa^{3} b_{l}-3 \kappa^{2} a_{l}\right) U_{\tilde{l}}^{\prime}=0
$$

Eqs. (73) and (74) reduce to the ODE

$$
\begin{aligned}
& b_{l} U_{l}^{(i v)}+6 \kappa^{2} b_{l} U_{l}^{\prime \prime}+\left(\kappa \alpha_{l}-\omega+\beta_{l}-3 \kappa^{4} b_{l}\right) U_{l} \\
& +2\left(c_{l}+d_{l}\right) U_{l}\left(U_{l}^{\prime}\right)^{2}+2\left(c_{l}+d_{l}\right) U_{l}^{2} U_{l}^{\prime \prime}+\left(\lambda_{l}+\mu_{l}+\zeta_{l}\right) U_{l}^{5}=0,
\end{aligned}
$$

with the constraints

$$
\begin{aligned}
& U_{\tilde{l}}=U_{l}, \\
& a_{l}=4 \kappa b_{l}, \\
& v=\alpha_{l}-8 \kappa^{3} b_{l} .
\end{aligned}
$$

Eq. (75) admits Eq. (27). Inserting Eqs. (27) and (11) into Eq. (75) gives rise to the following results:

Case 1:

$$
\begin{aligned}
A_{0}= & 0, A_{1}= \pm \sqrt{\frac{3 \kappa^{3} \alpha_{l}-3 \kappa^{2} \omega+3 \kappa^{2} \beta_{l}-10 \kappa \alpha_{l}+10 \omega-10 \beta_{l}}{6 c_{l}+6 \kappa^{4} d_{l}+18 \kappa^{2} c_{l}+18 \kappa^{2} d_{l}-12 c_{l}-12 d_{l}}}, \\
B_{1}= & 0, b_{l}=\frac{\kappa \alpha_{l}-\omega+\beta_{l}}{3\left(\kappa^{4}+3 \kappa^{2}-2\right)}, \\
\zeta_{l}= & -\frac{1}{\left(3 \kappa^{2}-10\right)^{2}\left(\kappa \alpha_{l}-\omega+\beta_{l}\right)} \times \\
& \left(108 \kappa^{6} c_{l}^{2}+216 \kappa^{6} c_{l} d_{l}+108 \kappa^{6} d_{l}^{2}+9 \kappa^{5} \alpha_{l} \lambda_{l}+9 \kappa^{5} \alpha_{l} \mu_{l}-9 \kappa^{4} \omega \lambda_{l}-9 \kappa^{4} \omega \mu_{l}\right. \\
& +9 \kappa^{4} \beta_{l} \lambda_{l}+9 \kappa^{4} \beta_{l} \mu_{l}+252 \kappa^{4} c_{l}^{2}+504 \kappa^{4} c_{l} d_{l}+252 \kappa^{4} d_{l}^{2}-60 \kappa^{3} \alpha_{l} \lambda_{l} \\
& -60 \kappa^{3} \alpha_{l} \mu_{l}+60 \kappa^{2} \omega \lambda_{l}+60 \kappa^{2} \omega \mu_{l}-60 \kappa^{2} \beta_{l} \lambda_{l}-60 \kappa^{2} \beta_{l} \mu_{l}-432 \kappa^{2} c_{l}^{2} \\
& -864 \kappa^{2} c_{l} d_{l}-432 \kappa^{2} d_{l}^{2}+100 \kappa \alpha_{l} \lambda_{l}+100 \kappa \alpha_{l} \mu_{l}-100 \omega \lambda_{l}-100 \omega \mu_{l}+100 \beta_{l} \lambda_{l} \\
& \left.+100 \beta_{l} \mu_{l}+144 c_{l}^{2}+288 c_{l} d_{l}+144 d_{l}^{2}\right) .
\end{aligned}
$$


Substituting Eqs. (79) and (12) into Eq. (27) causes the dark solitons

$$
\begin{aligned}
q(x, t) & = \pm \sqrt{\frac{3 \kappa^{3} \alpha_{1}-3 \kappa^{2} \omega+3 \kappa^{2} \beta_{1}-10 \kappa \alpha_{1}+10 \omega-10 \beta_{1}}{6 \kappa^{4} c_{1}+6 \kappa^{4} d_{1}+18 \kappa^{2} c_{1}+18 \kappa^{2} d_{1}-12 c_{1}-12 d_{1}}} \\
& \times \tanh \left(x-\left(\alpha_{1}-8 \kappa^{3} b_{1}\right) t\right) e^{i\left(-\kappa x+\omega t+\theta_{0}\right)} \\
r(x, t) & = \pm \sqrt{\frac{3 \kappa^{3} \alpha_{2}-3 \kappa^{2} \omega+3 \kappa^{2} \beta_{2}-10 \kappa \alpha_{2}+10 \omega-10 \beta_{2}}{6 \kappa^{4} c_{2}+6 \kappa^{4} d_{2}+18 \kappa^{2} c_{2}+18 \kappa^{2} d_{2}-12 c_{2}-12 d_{2}}} \\
& \times \tanh \left(x-\left(\alpha_{2}-8 \kappa^{3} b_{2}\right) t\right) e^{i\left(-\kappa x+\omega t+\theta_{0}\right)}
\end{aligned}
$$

and the singular solitons

with

$$
\begin{aligned}
q(x, t) & = \pm \sqrt{\frac{3 \kappa^{3} \alpha_{1}-3 \kappa^{2} \omega+3 \kappa^{2} \beta_{1}-10 \kappa \alpha_{1}+10 \omega-10 \beta_{1}}{6 \kappa^{4} c_{1}+6 \kappa^{4} d_{1}+18 \kappa^{2} c_{1}+18 \kappa^{2} d_{1}-12 c_{1}-12 d_{1}}} \\
& \times \operatorname{coth}\left(x-\left(\alpha_{1}-8 \kappa^{3} b_{1}\right) t\right) e^{i\left(-\kappa x+\omega t+\theta_{0}\right)} \\
r(x, t) & = \pm \sqrt{\frac{3 \kappa^{3} \alpha_{2}-3 \kappa^{2} \omega+3 \kappa^{2} \beta_{2}-10 \kappa \alpha_{2}+10 \omega-10 \beta_{2}}{6 \kappa^{4} c_{2}+6 \kappa^{4} d_{2}+18 \kappa^{2} c_{2}+18 \kappa^{2} d_{2}-12 c_{2}-12 d_{2}}} \\
& \times \operatorname{coth}\left(x-\left(\alpha_{2}-8 \kappa^{3} b_{2}\right) t\right) e^{i\left(-\kappa x+\omega t+\theta_{0}\right)}
\end{aligned}
$$

$$
\begin{aligned}
& \left(3 \kappa^{3} \alpha_{l}-3 \kappa^{2} \omega+3 \kappa^{2} \beta_{l}-10 \kappa \alpha_{l}+10 \omega-10 \beta_{l}\right) \\
& \times\left(6 \kappa^{4} c_{l}+6 \kappa^{4} d_{l}+18 \kappa^{2} c_{l}+18 \kappa^{2} d_{l}-12 c_{l}-12 d_{l}\right)>0 .
\end{aligned}
$$

Case 2:

$$
\begin{aligned}
& A_{0}=0, A_{1}=0 \\
& b_{l}=\frac{\kappa \alpha_{l}-\omega+\beta_{l}}{3 \kappa^{4}-6 \kappa^{2}-1}, \\
& B_{1}= \pm \sqrt{\frac{3 \kappa^{3} \alpha_{l}-3 \kappa^{2} \omega+3 \kappa^{2} \beta_{l}+5 \kappa \alpha_{l}-5 \omega+5 \beta_{l}}{3 \kappa^{4} c_{l}+3 \kappa^{4} d_{l}-6 \kappa^{2} c_{l}-6 \kappa^{2} d_{l}-c_{l}-d_{l}}}, \\
& \zeta_{l}=\frac{1}{\left(3 \kappa^{2}+5\right)^{2}\left(\kappa \alpha_{l}-\omega+\beta_{l}\right)} \times \\
& \left(54 \kappa^{6} c_{l}^{2}+108 \kappa^{6} c_{l} d_{l}+54 \kappa^{6} d_{l}^{2}-9 \kappa^{5} \alpha_{l} \lambda_{l}-9 \kappa^{5} \alpha_{l} \mu_{l}+9 \kappa^{4} \omega \lambda_{l}+9 \kappa^{4} \omega \mu_{l}-9 \kappa^{4} \beta_{l} \lambda_{l}\right. \\
& -9 \kappa^{4} \beta_{l} \mu_{l}-90 \kappa^{4} c_{l}^{2}-180 \kappa^{4} c_{l} d_{l}-90 \kappa^{4} d_{l}^{2}-30 \kappa^{3} \alpha_{l} \lambda_{l}-30 \kappa^{3} \alpha_{l} \mu_{l}+30 \kappa^{2} \omega \lambda_{l} \\
& +30 \kappa^{2} \omega \mu_{l}-30 \kappa^{2} \beta_{l} \lambda_{l}-30 \kappa^{2} \beta_{l} \mu_{l}-54 \kappa^{2} c_{l}^{2}-108 \kappa^{2} c_{l} d_{l}-54 \kappa^{2} d_{l}^{2}-25 \kappa \alpha_{l} \lambda_{l} \\
& \left.-25 \kappa \alpha_{l} \mu_{l}+25 \omega \lambda_{l}+25 \omega \mu_{l}-25 \beta_{l} \lambda_{l}-25 \beta_{l} \mu_{l}-6 c_{l}^{2}-12 c_{l} d_{l}-6 d_{l}^{2}\right) .
\end{aligned}
$$

Inserting Eqs. (84) and (12) into Eq. (27) yields the bright solitons

$$
\begin{aligned}
q(x, t) & = \pm \sqrt{\frac{3 \kappa^{3} \alpha_{1}-3 \kappa^{2} \omega+3 \kappa^{2} \beta_{1}+5 \kappa \alpha_{1}-5 \omega+5 \beta_{1}}{3 \kappa^{4} c_{1}+3 \kappa^{4} d_{1}-6 \kappa^{2} c_{1}-6 \kappa^{2} d_{1}-c_{1}-d_{1}}} \\
& \times \operatorname{sech}\left(x-\left(\alpha_{1}-8 \kappa^{3} b_{1}\right) t\right) e^{i\left(-\kappa x+\omega t+\theta_{0}\right)}
\end{aligned}
$$




$$
\begin{aligned}
r(x, t) & = \pm \sqrt{\frac{3 \kappa^{3} \alpha_{2}-3 \kappa^{2} \omega+3 \kappa^{2} \beta_{2}+5 \kappa \alpha_{2}-5 \omega+5 \beta_{2}}{3 \kappa^{4} c_{2}+3 \kappa^{4} d_{2}-6 \kappa^{2} c_{2}-6 \kappa^{2} d_{2}-c_{2}-d_{2}}} \\
& \times \operatorname{sech}\left(x-\left(\alpha_{2}-8 \kappa^{3} b_{2}\right) t\right) e^{i\left(-\kappa x+\omega t+\theta_{0}\right)}
\end{aligned}
$$

with

$$
\begin{aligned}
& \left(3 \kappa^{3} \alpha_{l}-3 \kappa^{2} \omega+3 \kappa^{2} \beta_{l}+5 \kappa \alpha_{l}-5 \omega+5 \beta_{l}\right) \\
& \times\left(3 \kappa^{4} c_{l}+3 \kappa^{4} d_{l}-6 \kappa^{2} c_{l}-6 \kappa^{2} d_{l}-c_{l}-d_{l}\right)>0
\end{aligned}
$$

and the singular solitons

$$
\begin{aligned}
q(x, t) & = \pm \sqrt{-\frac{3 \kappa^{3} \alpha_{1}-3 \kappa^{2} \omega+3 \kappa^{2} \beta_{1}+5 \kappa \alpha_{1}-5 \omega+5 \beta_{1}}{3 \kappa^{4} c_{1}+3 \kappa^{4} d_{1}-6 \kappa^{2} c_{1}-6 \kappa^{2} d_{1}-c_{1}-d_{1}}} \\
& \times \operatorname{csch}\left(x-\left(\alpha_{1}-8 \kappa^{3} b_{1}\right) t\right) e^{i\left(-\kappa x+\omega t+\theta_{0}\right)} \\
r(x, t)= & \pm \sqrt{-\frac{3 \kappa^{3} \alpha_{2}-3 \kappa^{2} \omega+3 \kappa^{2} \beta_{2}+5 \kappa \alpha_{2}-5 \omega+5 \beta_{2}}{3 \kappa^{4} c_{2}+3 \kappa^{4} d_{2}-6 \kappa^{2} c_{2}-6 \kappa^{2} d_{2}-c_{2}-d_{2}}} \\
& \times \operatorname{csch}\left(x-\left(\alpha_{2}-8 \kappa^{3} b_{2}\right) t\right) e^{i\left(-\kappa x+\omega t+\theta_{0}\right)}
\end{aligned}
$$

with

$$
\begin{aligned}
& \left(3 \kappa^{3} \alpha_{l}-3 \kappa^{2} \omega+3 \kappa^{2} \beta_{l}+5 \kappa \alpha_{l}-5 \omega+5 \beta_{l}\right) \\
& \times\left(3 \kappa^{4} c_{l}+3 \kappa^{4} d_{l}-6 \kappa^{2} c_{l}-6 \kappa^{2} d_{l}-c_{l}-d_{l}\right)<0 .
\end{aligned}
$$

Case 3:

$$
\begin{aligned}
A_{0}= & 0 \\
b_{l}= & \frac{8\left(\kappa \alpha_{l}-\omega+\beta_{l}\right)}{3\left(8 \kappa^{4}+6 \kappa^{2}-1\right)} \\
A_{1}= & \pm \sqrt{\frac{12 \kappa^{3} \alpha_{l}-12 \kappa^{2} \omega+12 \kappa^{2} \beta_{l}-10 \kappa \alpha_{l}+10 \omega-10 \beta_{l}}{24 \kappa^{4} c_{l}+24 \kappa^{4} d_{l}+18 \kappa^{2} c_{l}+18 \kappa^{2} d_{l}-3 c_{l}-3 d_{l}}} \\
B_{1}= & \sqrt{-\sqrt{\frac{12 \kappa^{3} \alpha_{l}-12 \kappa^{2} \omega+12 \kappa^{2} \beta_{l}-10 \kappa \alpha_{l}+10 \omega-10 \beta_{l}}{24 \kappa^{4} c_{l}+24 \kappa^{4} d_{l}+18 \kappa^{2} c_{l}+18 \kappa^{2} d_{l}-3 c_{l}-3 d_{l}}}} \\
\zeta_{l}= & -\frac{1}{4\left(6 \kappa^{2}-5\right)^{2}\left(\kappa \alpha_{l}-\omega+\beta_{l}\right)} \\
& \times\left(432 \kappa^{6} c_{l}^{2}+864 \kappa^{6} c_{l} d_{l}+432 \kappa^{6} d_{l}^{2}+144 \kappa^{5} \alpha_{l} \lambda_{l}\right. \\
& +144 \kappa^{5} \alpha_{l} \mu_{l}-144 \kappa^{4} \omega \lambda_{l}-144 \kappa^{4} \omega \mu_{l}+144 \kappa^{4} \beta_{l} \lambda_{l}+144 \kappa^{4} \beta_{l} \mu_{l}+252 \kappa^{4} c_{l}^{2} \\
& +504 \kappa^{4} c_{l} d_{l}+252 \kappa^{4} d_{l}^{2}-240 \kappa^{3} \alpha_{l} \lambda_{l}-240 \kappa^{3} \alpha_{l} \mu_{l}+240 \kappa^{2} \omega \lambda_{l}+240 \kappa^{2} \omega \mu_{l} \\
& -240 \kappa^{2} \beta_{l} \lambda_{l}-240 \kappa^{2} \beta_{l} \mu_{l}-108 \kappa^{2} c_{l}^{2}-216 \kappa^{2} c_{l} d_{l}-108 \kappa^{2} d_{l}^{2}+100 \kappa \alpha_{l} \lambda_{l}+100 \kappa \alpha_{l} \mu_{l} \\
& \left.-100 \omega \lambda_{l}-100 \omega \mu_{l}+100 \beta_{l} \lambda_{l}+100 \beta_{l} \mu_{l}+9 c_{l}^{2}+18 c_{l} d_{l}+9 d_{l}^{2}\right) .
\end{aligned}
$$

Substituting Eqs. (89) and (12) into Eq. (27) yields the combo singular solitons 


$$
\begin{aligned}
q(x, t) & = \pm \sqrt{\frac{12 \kappa^{3} \alpha_{1}-12 \kappa^{2} \omega+12 \kappa^{2} \beta_{1}-10 \kappa \alpha_{1}+10 \omega-10 \beta_{1}}{24 \kappa^{4} c_{1}+24 \kappa^{4} d_{1}+18 \kappa^{2} c_{1}+18 \kappa^{2} d_{1}-3 c_{1}-3 d_{1}}} \\
& \times\left\{\operatorname{coth}\left(x-\left(\alpha_{1}-8 \kappa^{3} b_{1}\right) t\right)+\operatorname{csch}\left(x-\left(\alpha_{1}-8 \kappa^{3} b_{1}\right) t\right)\right\} \\
& \times e^{i\left(-\kappa x+\omega t+\theta_{0}\right)}, \\
r(x, t) & = \pm \sqrt{\frac{12 \kappa^{3} \alpha_{2}-12 \kappa^{2} \omega+12 \kappa^{2} \beta_{2}-10 \kappa \alpha_{2}+10 \omega-10 \beta_{2}}{24 \kappa^{4} c_{2}+24 \kappa^{4} d_{2}+18 \kappa^{2} c_{2}+18 \kappa^{2} d_{2}-3 c_{2}-3 d_{2}}} \\
& \times\left\{\operatorname{coth}\left(x-\left(\alpha_{2}-8 \kappa^{3} b_{2}\right) t\right)+\operatorname{csch}\left(x-\left(\alpha_{2}-8 \kappa^{3} b_{2}\right) t\right)\right\} \\
& \times e^{i\left(-\kappa x+\omega t+\theta_{0}\right)},
\end{aligned}
$$

with

$$
\begin{aligned}
& \left(12 \kappa^{3} \alpha_{l}-12 \kappa^{2} \omega+12 \kappa^{2} \beta_{l}-10 \kappa \alpha_{l}+10 \omega-10 \beta_{l}\right) \\
& \times\left(24 \kappa^{4} c_{l}+24 \kappa^{4} d_{l}+18 \kappa^{2} c_{l}+18 \kappa^{2} d_{l}-3 c_{l}-3 d_{l}\right)>0 .
\end{aligned}
$$

\section{Conclusion}

In the present study, we have recovered the solutions in the forms of bright, dark and singular optical solitons for the cases of third- and fourth-order dispersive reflectivities, as opposed to a common norm of dispersive reflectivity with the $\mathrm{CD}$. This very concept and the corresponding results have been reported and analyzed for the first time in the field of nonlinear optics. Hence, these results are truly novel. The results obtained in this work and the new technicalities developed by us would enhance both a general soliton science and a dynamics of solitons propagating through optical fibres across intercontinental distances. The foundation stones for such a new concept are thus grounded.

What needs to be done next is expanding and extending the ideas mentioned above to various additional scenarios. The latter would include retrieval of the conservation laws within the model, addressing the model with fractional temporal evolutions and studying the appropriate model with stochasticity (see, e.g., the stochastic coefficients introduced in Refs. [27-30]). In addition to the analytical approaches, the models developed in the present work can be handled numerically. Moreover, the known Adomian and Laplace-Adomian decomposition schemes, the finite-element approach and some other techniques can be implemented. Such research activities are now in progress.

\section{Disclosure}

The authors declare no conflict of interest.

\section{References}

1. Ahmed T and Atai J, 2017. Bragg solitons in systems with separated nonuniform Bragg grating and nonlinearity. Phys. Rev. E. 96: 032222.

2. Ahmed T and Atai J, 2019. Soliton-soliton dynamics in a dual-core system with separated nonlinearity and nonuniform Bragg grating. Nonlinear Dyn. 97: 1515- 1523.

3. Akter A and Atai J, 2020. Interactions of gap solitons in coupled Bragg gratings with cubicquintic nonlinearity and dispersive reflectivity. In Proceedings of the 8th International Conference on Photonics, Optics and Laser Technology. 1: 22-25. 
4. Cao H, Shu X, Atai J, Gbadebo A, Xiong B, Fan T, Tang H S, Yang W and Yu Y, 2014. Optimally-designed single fiber Bragg grating filter scheme for RZ-OOK/DPSK/DQPSK to NRZ-OOK/DPSK/DQPSK format conversion. Opt. Express. 22 (25): 30442-30460.

5. Cao H, Shu X, Atai J, Zuo J, Xiong B, Shen F and Cheng J, 2015. Fiber Bragg grating based notch filter for bit-rate-transparent NRZ to PRZ format conversion with two-degree-offreedom optimization. J. Opt. 17: 025702.

6. Chowdhury S A M S and Atai J, 2017. Moving Bragg grating solitons in a semilinear dualcore system with dispersive reflectivity. Sci. Rep. 7: 4021.

7. Islam M J and Atai J, 2014. Stability of gap solitons in dual-core Bragg gratings with cubicquintic nonlinearity. Laser Phys. Lett. 12: 015401.

8. Islam M S, Sultana J, Dorraki M, Atai J, Islam M R, Dinovitser A, Ng B W H and Abbott D, 2018. Low loss and low dispersion hybrid core photonic crystal fiber for terahertz propagation. Photon. Netw. Commun. 35: 364-373.

9. Islam M J and Atai J, 2019. Dynamics of colliding counterpropagating solitons in coupled Bragg gratings with cubic-quintic nonlinearity. J. Mod. Opt. 66: 1498-1505.

10. Biswas A, Ekici M, Sonmezoglu A and Belic M, 2019. Solitons in optical fiber Bragg gratings with dispersive reflectivity by extended trial function method. Optik. 182: 88-94.

11. Biswas A, Vega-Guzman J, Mahmood M F, Khan S, Zhou Q, Moshokoa S P and Belic M, 2019. Solitons in optical fiber Bragg gratings with dispersive reflectivity. Optik. 182: 119123.

12. Biswas A, Ekici M, Sonmezoglu A and Belic M R, 2019. Optical solitons in fiber Bragg gratings with dispersive reflectivity for parabolic law nonlinearity by extended trial function method. Optik. 183: 595-601.

13. Biswas A, Vega-Guzman J, Mahmood M F, Ekici M, Zhou Q, Moshokoa S P and Belic M R, 2019. Optical solitons in fiber Bragg gratings with dispersive reflectivity for parabolic law nonlinearity using undetermined coefficients. Optik. 185: 39-44.

14. Biswas A, Ekici M, Sonmezoglu A, Belic M R, 2019. Optical solitons in fiber Bragg gratings with dispersive reflectivity for quadratic-cubic nonlinearity by extended trial function method. Optik. 185: 50-56.

15. Biswas A, Sonmezoglu A, Ekici M, Alshomrani A S and Belic M R, 2019. Optical solitons in fiber Bragg gratings with dispersive reflectivity for cubic-quintic-septic nonlinearity by extended trial function. Optik. 194: 163020.

16. Biswas A, Sonmezoglu A, Ekici M, Alshomrani A S and Belic M R, 2019. Optical solitons in fiber Bragg gratings with dispersive reflectivity for parabolic-nonlocal combo nonlinearity by extended trial function. Optik. 195: 163146.

17. Darwish A, Abo El-Dahab E, Ahmed H, Arnous A H, Ahmed M S, Biswas A, Guggilla P, Yildirim Y, Mallawi F and Belic M R, 2020. Optical solitons in fiber Bragg gratings via modified simple equation. Optik. 203: 163886.

18. Kudryashov N A, 2020. Periodic and solitary waves in optical fiber Bragg gratings with dispersive reflectivity. Chin. J. Phys. 66: 401-405.

19. Yildirim Y, Biswas A, Khan S, Guggilla P, Alzahrani A K and Belic M R, 2021. Optical solitons in fiber Bragg gratings with dispersive reflectivity by sine-Gordon equation approach. Optik. 237: 166684.

20. Zayed E M E, Shohib R M A, Biswas A, Yildirim Y, Mallawi F and Belic M R, 2019. Chirped and chirp-free solitons in optical fiber Bragg gratings with dispersive reflectivity

Ukr. J. Phys. Opt. 2021, Volume 22, Issue 4 
having parabolic law nonlinearity by Jacobi’s elliptic function. Results Phys. 15: 102784.

21. Zayed E M E, Alngar M E M, Biswas A, Triki H, Yildirim Y and Alshomrani A S, 2020. Chirped and chirp-free optical solitons in fiber Bragg gratings with dispersive reflectivity having quadratic-cubic nonlinearity by sub-ODE approach. Optik. 203: 163993.

22. Zayed E M E, Alngar M E M, Biswas A, Ekici M, Triki H, Alzahrani A K and Belic M R, 2020. Chirped and chirp-free optical solitons in fiber Bragg gratings having dispersive reflectivity with polynomial form of nonlinearity using sub-ODE approach. Optik. 204: 164096

23. Zayed E M E, Shohib R M A, Biswas A, Ekici M, Triki H, Alzahrani A K and Belic M R, 2020. Optical solitons with fiber Bragg gratings and dispersive reflectivity having parabolicnonlocal combo nonlinearity via three prolific integration architectures. Optik. 208: 164065.

24. Zayed E M E, Shohib R M A, Biswas A, Gonzalez-Gaxiola O, Yildirim Y, Alzahrani A K and Belic M R, 2020. Optical solitons in fiber Bragg gratings with generalized anti-cubic nonlinearity by extended auxiliary equation. Chin. J. Phys. 65: 613-628.

25. Zayed E M E, Alngar M E M, El-Horbaty M, Biswas A, Alshomrani A S, Khan S, Ekici M and Triki H, 2020. Optical solitons in fiber Bragg gratings having Kerr law of refractive index with extended Kudryashov's method and new extended auxiliary equation approach. Chin. J. Phys. 66: 187-205.

26. Zayed E M E, Alngar M E M, Biswas A, Ekici M, Alzahrani A K and Belic M R, 2020. Chirped and chirp-free optical solitons in fiber Bragg gratings with Kudryashov's model in presence of dispersive reflectivity. J. Commun. Technol. Electron. 65: 1267-1287.

27. Zhou Q, Liu L, Zhang H, Wei C, Liu J, Yu H and Biswas A, 2015. Analytical study of Thirring optical solitons with parabolic law nonlinearity and spatio-temporal dispersion. Eur. Phys. J. Plus. 130: 138.

28. Zhou Q, Zhu Q, Bhrawy A H and Biswas A, 2015. Combined optical solitons with nonlinear dispersion and spatio-temporal dispersion. Optoelectron. Adv. Mater. Rapid Commun. 9: 1-4.

29. Zhou Q, Liu L, Zhang H, Mirzazadeh M, Bhrawy A H, Zerrad E, Moshokoa S and Biswas A, 2016. Dark and singular optical solitons with competing nonlocal nonlinearities. Opt. Appl. 46: $79-86$.

30. Zhou Q and Biswas A, 2017. Optical solitons in parity-time-symmetric mixed linear and nonlinear lattice with non-Kerr law nonlinearity. Superlattices Microstruct. 109: 588-598.

Yakup Yıldırım, Anjan Biswas, Padmaja Guggilla, Salam Khan, Hashim M. Alshehri and Milivoj R. Belic. 2021. Optical solitons in fibre Bragg gratings with third- and fourth-order dispersive reflectivities. Ukr.J.Phys.Opt. 22: 239 - 254. doi: 10.3116/16091833/22/4/239/2021

Анотація. Вперше в нелінійній оптиці розглянуто кубічно-квартичні солітони, які з'являються у волоконних бреггівських тратках з дисперсійною відбивною здатністю для чотирьох різних випадків структур з нелінійним показником заломлення. Із прийнятої нами схеми інтегрування, яка є підходом рівняння синус-Гордона, випливає повний спектр одиничних солітонів, разом із деякими розмежованими солітонами. 